\title{
Wireless charging system using spectral energy harvesting technique
}

\author{
Chandrasekharan Nataraj, Mahsoom Raseen Abdul Careem, Ravi Lakshmanan, \\ Sathish Kumar Selvaperumal, Raed Abdulla \\ Faculty of Computing, Engineering and Technology, Asia Pacific University (APU), Malaysia
}

\begin{abstract}
Article Info
Article history:

Received Mar 14, 2018

Revised Jul 23, 2018

Accepted Jan 12, 2019

\section{Keywords:}

Charge pump circuit

Patch array antenna

SDR sharp

Spectral energy harvesting

Wireless charging

ABSTRACT

This paper presented the energy harvester circuit to enable wireless charging method. The wireless RF spectrum is abundant and potent in the public locations, which can be used for energy harvesting purpose. This is supported by the fact that RF signals are ubiquitous and do not require propagation media of any sort. The system is developed using optimal antenna, matching circuit and charge pump circuit. The $2 \times 2$ array type patch antenna operated at $924 \mathrm{MHz}$ is designed and simulated using High Frequency Simulator. The antenna works as a transducer to capture and measure the RF signals from freely available energy source. The field study was conducted at three different highly populated locations such as place of education, place of residence, and place of transport using Software Defined Radio (SDR). The respective acquired RF energy values are $-10.45 \mathrm{dBFS}$ (morning), -10.45 dBFS (noon), and -13.33 dBFS (night) at the place of transport for the demonstration purpose. The antenna parameters study also conducted and proved that the antenna gain is good which is greater than $6.6 \mathrm{~dB}$ and also return loss greater than $-43 \mathrm{~dB}$. In addition, charge pump circuit is designed and shown to be capable of boosting the voltage more than $2.5 \mathrm{~V}$ easily at the different frequencies for wireless charging purpose.
\end{abstract}

Copyright @ 2019 Institute of Advanced Engineering and Science. All rights reserved.

\section{Corresponding Author:}

Chandrasekharan Nataraj, Faculty of Computing, Engineering and Technology,

Asia Pacific University (APU),

57000 Kuala Lumpur, Malaysia.

Email: chander@apu.edu.my

\section{INTRODUCTION}

Charging of electronic gadgets have been evolved from directly plugging the charger to the source with the usage of micro-USB cables to supply the power. The trend in the advancement of power banks for movable or flexible charging redirected our interest towards wireless pad and cable-less charging. Nowadays cable-less charging systems are in high demand due to increased usage of portable tiny devices [1]. In the near future, cables are likely to be non-existent for charging and the use of wireless pads would emerge into trend. It is the cable-less technique to transfer power between two remotely located coils or antennas. Commonly, wireless power transfer has been attained through three techniques such as inductive coupling, capacitive coupling and resonant charging [2].

Inductively coupled transfer technique is quite popular in which inductive coils and electrodes are employed for wireless charging [3]. A capacitive coupling transfers pulses from one portion of the circuit to another using a coupling capacitor connected in series between two circuits. This process offers high impedance to the DC voltage and low resistance for AC signals [4]. Resonance coupling also uses the inductive principle but it is able to transfer maximum power at the resonance frequency. A resonantly 
coupled inductive power transfer scheme is typically adopted for the Radio Frequency (RF) wireless power transfer applications such as biomedical implants, RFID tags and mobile phone charging [5-7].

WPT for mobile phones was achieved by the means of electro-magnetic induction that harvest the energy from a current source [8]. The system is limited due to the requirement of a source, which eventually limits the distance of charging, resulting in non-feasible and uncomfortable use of mobile phones while charging. As a solution to this, energy harvesting was determined to be feasible for its capability of harnessing surrounding sources [9]. As such the solar energy harvesting technique was proposed due to its renewable and unreserved aspects [10]. But the success of solar based energy harvesting will be contingent with presence of light source, place, weather and time, i.e., limited in terms of availability and accessibility. Subsequently, energy harvesting was explored about ambient (freely available) energy sources, which is frequently available unlike wind, solar, hydro etc. A thermoelectric energy harvesting was proposed to harvest the power from the human body heat for mobile charging [11]. The human body was determined to generate very low voltage resulting in non-viability for charging purposes. Similar results were observed using other harvesting methods by means of mobile phone headsets [1] and Bluetooth technology [12].

The RF spectrum based study was conducted in a city center and samples acquired have shown proficient results of providing higher efficiency of power transfer [13]. This technique is observed to be applied for Radio Frequency Identification (RFID) for battery-free programming platforms and also in UHF RFID systems [14] achieving 16-30\% efficiency. To attain higher power transfer efficiency from 2 G Global System for Mobile communications (GSM) spectrum due to its abundant usage, the charge-pump electronic circuit was designed and implemented [15]. As 2G GSM limits to one particular band of spectrum so that this kind of harvested system can produce a range of higher energy harvesting efficiency rates and signal strength using Omni-directional antennas [16]. When compared to conventional monopole antennas, micro strip patch has numerous advantages so that it is adopted for wide range of applications such as mobile communication and satellite aircrafts [17].

In a nutshell, inductive and capacitive coupling techniques were considered viable for wireless charging. However self-limitation of these techniques is the requirement of input supply source. It makes the system to be a bit costlier and amount of power transferred is restricted with the ability of input supply source. The RF based energy harvesting is being considered as a standby technique to accomplish the wireless power transfer between remotely located circuits without input source. The principle idea behind this energy harvesting scheme is conducted using ambient (freely available) RF energy field in the free space. RF pulses are generated from ambient energy using omnidirectional antenna and further processed for harvesting energy without wires.

This paper presents radio frequency antenna principle to charge portable electronic gadgets using ambient energy scavenging technique. The feasibility and consistency of the existing RF spectrum for energy harvesting is investigated. An optimal antenna was designed to capture RF signals from ambitious energy source. A charge-pump circuit is designed to process captured signals. The necessary parameters for the design completion were executed using Ansoft HFSS software with Designer suite, and Diptrace to attain simulation results to verify the product feasibility.

This paper is organized as follows: Section 2 describes the system design involved with RF energy harvesting and also presented the necessary design calculations. Experimental results based on the strength of the ambient energy field study, antenna design simulations, charge-pump simulation and impedance matching are explored in the Section 3. At the end, major conclusions of this work are highlighted.

\section{SYSTEM DESIGN AND ITS CALCULATIONS}

The proposed system consists two major sections such as RF energy harvesting section and charge pump circuit. Figure 1 shows the block diagram of RF energy harvesting system. The first terminal device is the antenna which is used to collect the RF pulses from the freely available RF field at the populated places. The antenna is used to convert RF signals into AC pulses on the frequency spectrum that is harvested. The collected RF signal is not much enough to supply the power to the load and hence needed to be enhanced well. A charge pump circuit is used here to enhance the captured signal. It is an electronic circuit that consists of rectifier and voltage doubler to generate the sufficient dc signal to power up the load. The rectified signal is passed into the IC 756 based voltage doubler circuit to step-up the voltage up to $2.4 \mathrm{~V}$ which is the minimum requirement for practical usage.

The charge pump contains a Cockroft-Walton multiplier circuit which is used to boost the signal based on the assigned number of stages. As the voltage may not be stable as expected and might fluctuate, MAX 756 IC was implemented to reduce the fluctuation and make sustained power at the charge port. It also contains the matching circuit for impedance balancing. The frequencies proposed to harvest were $2 \mathrm{G}$ GSM (850-950 MHz), 3G GSM (2.1 GHz), Wi-Fi band $1(2.45 \mathrm{GHz})$ and Wi-Fi band $2(5 \mathrm{GHz})$ as a result of the 
overwhelming usage due to abundance in customers. In practice, patch antennas were considered for harvesting due to its omnidirectional and compact feature [13]. But the gain of these antennas are not sufficient to harvest the RF signals from the free space energy. Hence a $2 \times 2$ Array antenna is designed and proposed as conjoined patches to attain higher gain for better signal reception. Therefore patch array is designed and simulated for good signal strength feasibility. Figure 2 show Patch antenna layout.

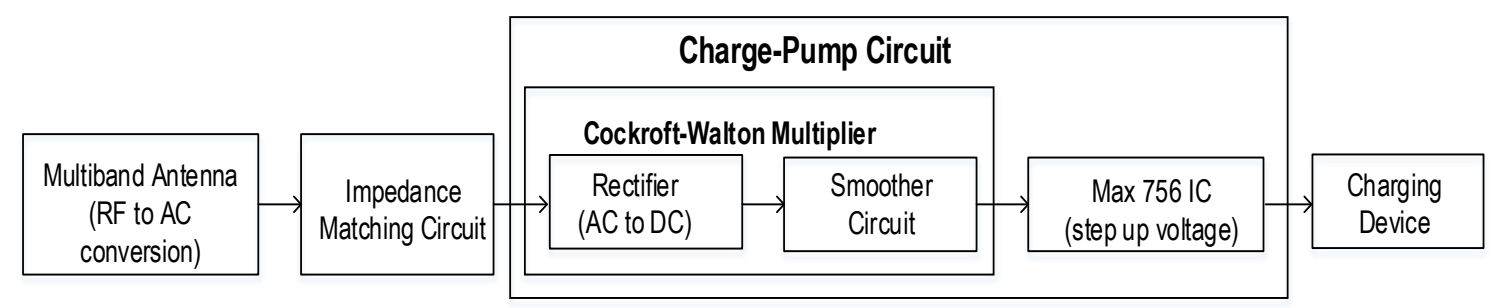

Figure 1. Block diagram of energy harvester circuit

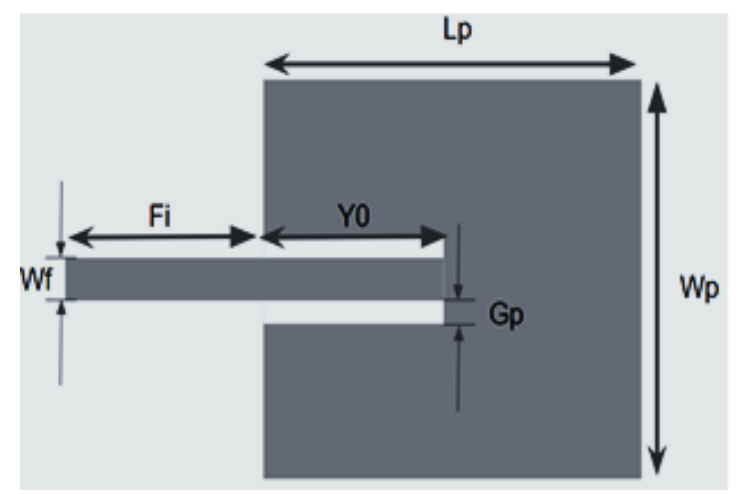

Figure 2. Patch antenna layout

\subsection{Patch Array Antenna Design}

Here the variables Wp and Lp represents length and width of the antenna structure. The variable Er represents dielectric constant and $\mathrm{H}$ represents substrate thickness. The antenna structure can be determined by using conventional antenna design formula [18]. However, single patch antenna does not have enough gain to collect and process the RF signal from the freely available spectrum. Hence the micro strip patch antenna array is designed and simulated to harvest the maximum signal to supply power to the resistive loads. The array antenna model is shown in Figure 3 displaying the parameters used for each division. A $2 \times 2$ array antenna was constructed with 4 rectangular patch antennas. The design calculations and parameter settings of array patch antennas were similar to what is described in the previous section of single patch antenna design. However the feed impedance of $100 \Omega$ is used in the array antenna instead of $50 \Omega$. In the case of array antennas in which the 4 patch antennas are to be fed, the $100 \Omega$ feeder lines are transformed into $50 \Omega$ ones, by using a quarter wave transformer with a quarter wave length and matched impedance of 70.7 ohms $\left(Z_{0}=\sqrt{50 \times 100}=70.7 \Omega\right.$. $)$. Antenna Design Parameters for Simulation as shown in Table 1 .

Table 1. Antenna Design Parameters for Simulation

\begin{tabular}{cc}
\hline Parameters & Value \\
\hline Dielectric constant (Er) & 4.4 \\
Dielectric substrate thickness (h) & $0.8 \mathrm{~mm}$ \\
Frequency (in GHz) & $0.9 / 2.1 / 2.45 / 5$ \\
Input impedance (z) & $50 \Omega$ \\
\hline
\end{tabular}




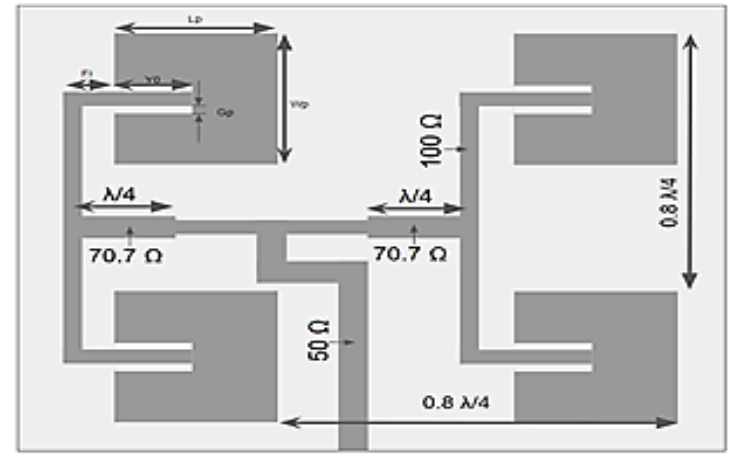

Figure 3. 2x2 Array antenna layout

The $100 \Omega$ lines is again converted back to $50 \Omega$ ones which are then split again to feed the individual patch antennas. Thus, the feed of each patch is $100 \Omega$. In addition, each patch is fed by the feeders of the same length which ensures feeding with same phase into each patch. The clearance between each patch was set to $0.8 \lambda$, which minimizes the interference in the radiation of each patch.

\subsection{Charge Pump Circuit Design}

The Cockroft-Walton multiplier was found to be the most suitable voltage multiplier circuit and also commonly adopted for amplification circuitries [19]. Figure 4 shows the PCB layout of multiplier circuit, which consists of cascaded stages of diodes and capacitors that helps to rectify and boost the voltage harvested by the antenna at the input. The capacitor calculation is used to determine the number of stages and output voltage. Table 2 show capacitance design for different frequency bands.

The expression for the voltage ripple and voltage drop is given as follows:

$$
\Delta V=\frac{I_{L}}{f_{C}} \times \frac{N(N+1)}{2}
$$

Where $\mathrm{N}$ is the number of capacitors used.

The optimum number of stages is calculated by the following formula,

$$
\begin{aligned}
& n_{\text {opt }}=\sqrt{\frac{V_{\text {max }} f C}{l}} \\
& V_{\text {out }}=V_{\text {in }} \times \sqrt{2} \times n
\end{aligned}
$$

The output voltage would be, $V_{\text {out }}=V_{\text {max }}-\Delta V+\delta V$

$$
V_{O}=n\left\{2 V_{\text {max }}-\frac{I}{6 f C}\left(4 n^{2}+3 n-1\right)+\frac{l}{2 f C}(n+1)\right\}
$$

By solving the above (6),

$$
16 V_{0}^{3} f^{2} C^{2}+\left(\left(16 V_{\max ^{2}}-9 V_{0}\right) f I\right) C+4 V_{\max } I=0
$$

This is in the form of quadratic nature and can be solved using traditional formula as follows:

$$
x=\frac{-b \pm \sqrt{\left(b^{2}-4 a c\right)}}{2 a}
$$

Rewriting this equation by subjecting the capacitance $\mathrm{C}$ from previous equation, 


$$
C=\frac{-\left(\left(16 V^{2} \max -9 V_{0}\right) f l\right) \pm \sqrt{\left(\left(\left(16 V^{2}{ }_{\max }-9 V_{0}\right) f I\right)^{2}-4\left(16 V^{3} \max ^{2}\right)\left(4 V_{\max } I\right)\right)}}{2\left(16 V^{2} \max -9 V_{0}\right) f I} .
$$

Table 2. Capacitance Design for Different Frequency Bands

\begin{tabular}{ccc}
\hline Name of Band & Frequency & Capacitance \\
\hline 2G GSM & $900 \mathrm{MHz}$ & $6.8 \mathrm{nF} / 33 \mathrm{pF}$ \\
3G GSM & $2.1 \mathrm{GHz}$ & $2.7 \mathrm{nF} / 15 \mathrm{pF}$ \\
Wi-Fi Band 1 & $2.45 \mathrm{GHz}$ & $2.3 \mathrm{nF} / 13 \mathrm{pF}$ \\
Wi-Fi Band 2 & $5 \mathrm{GHz}$ & $1.1 \mathrm{nF} / 6 \mathrm{pF}$ \\
\hline
\end{tabular}

\subsection{Matching Circuit Design}

The Matching circuit ensures that there is minimal reflection and maximum power transfer from the antenna to the core circuit. A simple RLC circuit is used as a matched circuit between source and load for achieving better performance. Here array antenna and charge pump circuit are being considered as source and load respectively.

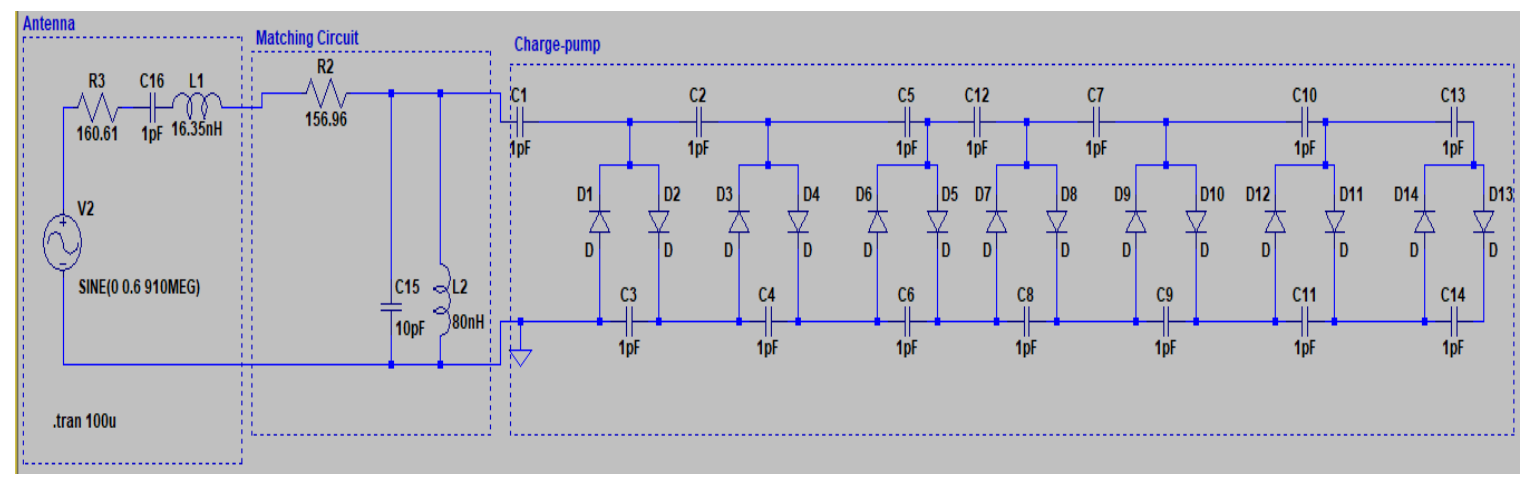

Figure 4. Charge pump circuit PCB layout

\subsection{Energy Harvester Design}

The voltage generated at the next stage of charge pump circuit is usually referred as RF harvester. Practically Decibels relative to Full Scale (dBFS) is used to measure the field intensity and also maximum input power rating of the Software Defined Radio (SDR) is $10 \mathrm{dBm}$, i.e., $10 \mathrm{dBm}$ is the full scale deflection (0 dBFS) for the SDR according to the Register Transfer Level-Software Defined Radio (RTL-SDR) datasheet [20] and hence $10 \mathrm{dBm}=0 \mathrm{dBFs}$ and $0 \mathrm{dBm}=-30 \mathrm{~dB}$. The feasibility study conducted for 924 MHz using the SDR was read to be $-10 \mathrm{dBFS}$ so that the scaling can be expressed as follows:

$$
\begin{aligned}
& 0-10 d B F S=10 d B m-10 d B m \\
& -10 d B F S=0 d B m
\end{aligned}
$$

The power equation is given in terms of $\mathrm{dBm}$ as $P d B m=10 \log P_{m W}$

$$
P_{m W}=10^{\frac{P d B m}{10}} m W \text { and hence } P_{m W}=1 m W
$$

The power according to the Ohm's law,

$$
P=\frac{V^{2}}{R} \text { and } V=\sqrt{P R}
$$


In the (12), the variable $\mathrm{R}$ refers to an achieved radiation resistance of the antenna. Similarly in another case of $920 \mathrm{MHz}$ the SDR had read up to 6dBFS which is justified using the following equations.

$$
\begin{aligned}
& 0-6 d B F S=10 d B m-6 d B m \\
& -6 d B F S=4 d B m \\
& 4 d B m=10 \log P_{m W} \text { and hence } P_{m W}=2.512 \mathrm{~mW} \\
& V=\sqrt{2.512 \times 10^{-3} \times 160.61}=0.6 \mathrm{~V}
\end{aligned}
$$

The results $-10 \mathrm{dBFS}$ and $-6 \mathrm{dBFS}$ are proven to be achieved in Table 4 under Section 3 and hence these values are used in this system design. Voltages achieved for other dBFS values were computed and listed in Table 3 as well. It is maintained with the use of a uniform input voltage $0.6 \mathrm{~V}$ for the simulation to compute harvested voltage of the developed system [7]. In addition, a MAX 756 Integrated Circuit (IC) was implied as a voltage booster to stabilize and condition the harvested pulses.

\section{TESTING AND RESULTS ANALYSIS}

There were four different tests such as the measurement of spectrum availability, measurement of gain and return loss of antenna, matching circuit and output voltage measurement of charge-pump circuit were conducted to ensure the system feasibility and efficiency. In order to design the harvester circuit, the investigation and identification of freely available RF spectrum is the most significant part. The RTL SDR is being used to study the spectrum in general areas. SDR is a device that can be plugged into any computer and can analyze the surrounding signals of the spectrum with the help of SDR Sharp software, which is basically a platform to read the SDR device. In this work, the field study was conducted at three different locations which is highly populated, such as place of education (Asia Pacific University, Bukit Jalil), place of residence (One South apartments, Serdang) and place of public transport (LRT station, Bukit Jalil). To explore the concept of spectrum detection, the detected RF spectrum and its energy levels at one of the aforementioned locations (Bukit Jalil) using 2G GSM band are displayed in Figure 5.

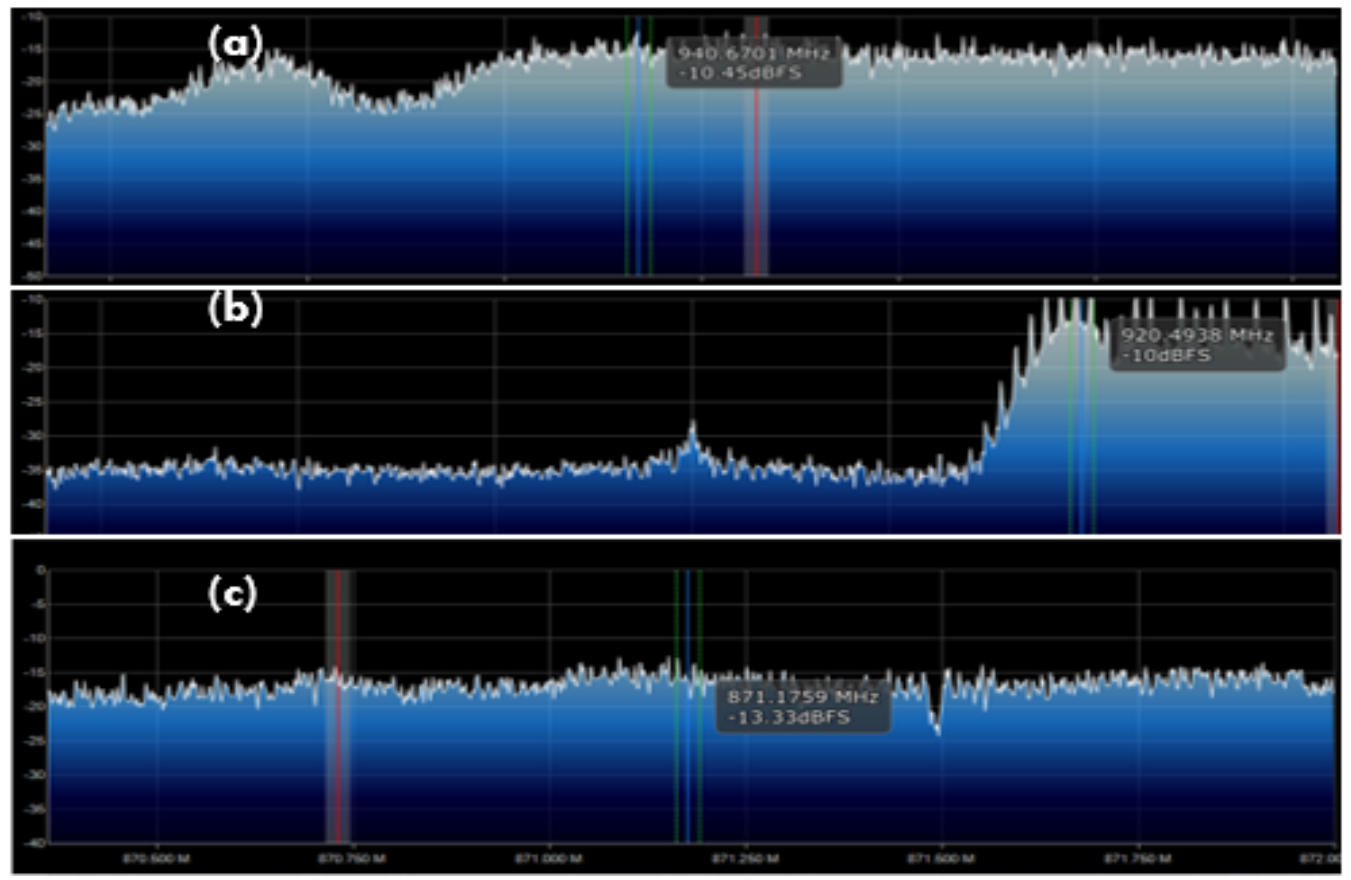

Figure 5. RF Spectrum test results at 2G GSM frequencies (850-950 MHz) for APU Bukit Jalil at (a) morning, (b) noon (c) night 
The reason for the chosen location was to identify places where usage of cell phones is in abundant, so that it results in higher RF signals in the surrounding. The field study has been conducted for three times in a day such as morning, noon and night time to observe the spectrum consistency. The RTL SDR had a limit of analyzing up to $2 \mathrm{GHz}$, therefore the study was proceeded for an additional band of Ultra High Frequency (UHF), in which the frequency of $550 \mathrm{MHz}$ used for television reception. Other frequencies used in this system are 2G GSM and 3G GSM for measurements and validations.

The respective acquired RF energy values are -10.45 dBFS (morning), -10.45 dBFS (noon), and 13.33 dBFS (night). The same concept is used at other locations and their relative RF signals are detected, which are shown in the Table. 3. It is observed that the spectrum was analyzed in three different locations at three different times to ensure the consistency of signal strength. In the overall measurements performed with three different locations and time durations, the best voltages harvested are tabulated in Table 4. Figures 6 and 7 shows the simulated results of patch array antennas such as return loss, gain and impedance diagrams from Ansoft HFSS with the frequency of $924 \mathrm{MHz}$. In practice, the minimum requirement of return loss is $10 \mathrm{~dB}$ for the effective performance [10]. Return losses were clarified to be reaching below $-20 \mathrm{~dB}$ which is being greater than the required return loss. Similarly, obtained gain is significantly appropriate in terms of antenna geometry and its structure.

Table 3. Field Test Results with Different Frequency Bands at Different Locations

\begin{tabular}{cccccc}
\hline \multirow{2}{*}{$\begin{array}{c}\text { Name } \\
\text { of Band }\end{array}$} & Frequency & \multirow{2}{*}{ Location } & \multicolumn{3}{c}{ Signal Strength (dBFS) } \\
\cline { 4 - 6 } & Band & & Morning & Day & Night \\
UHF & $450-$ & APU & -12.27 & -12.42 & -5.45 \\
& $550 \mathrm{MHz}$ & One South & -10.45 & -10 & 0 \\
& & LRT & -2.73 & -1.14 & -12 \\
\multirow{4}{*}{$2 \mathrm{G}$} & $850 \mathrm{MHz}-$ & APU & -10.45 & -10 & -6.14 \\
& $950 \mathrm{MHz}$ & One South & -10.61 & -11.97 & -13.33 \\
& & LRT & -9.35 & -10.25 & -7.45 \\
$3 \mathrm{G}$ & \multirow{2}{*}{$1.8-2 \mathrm{GHz}$} & APU & -10 & -10.15 & 0 \\
& & One South & -11.67 & -5.45 & -11.82 \\
& & LRT & -11.9 & -9.6 & -10.42 \\
\hline
\end{tabular}

Table 4. Output Voltage Generated by Antenna for Various Received Powers

\begin{tabular}{ccc}
\hline Power received (dBFS) & Power received $(\mathrm{dB})$ & Voltage $(\mathrm{V})$ \\
\hline 0 & -20 & 1.289 \\
-1.14 & -21.14 & 1.1128 \\
-2.73 & -22.73 & 0.9266 \\
-5.45 & -25.45 & 0.6675 \\
-6.31 & -26.31 & 0.6136 \\
\hline
\end{tabular}

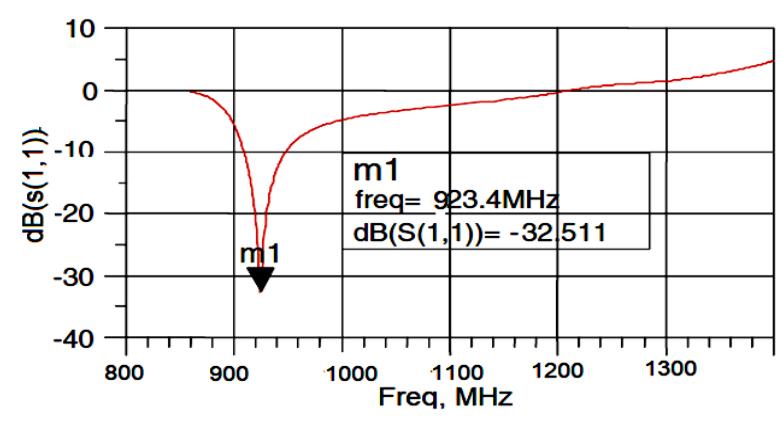

Figure 6. Simulation results for return loss in the array patch antenna design

The ability of the antenna is the most significant and vital parameter to harvest the energy and evaluate the system performance. As it is mentioned that $2 \times 2$ array type patch antenna is used as the input device to absorb the radio frequency energy from freely available ambitious RF field. The simulation results of developed antenna for the purpose of energy harvesting is presented. 


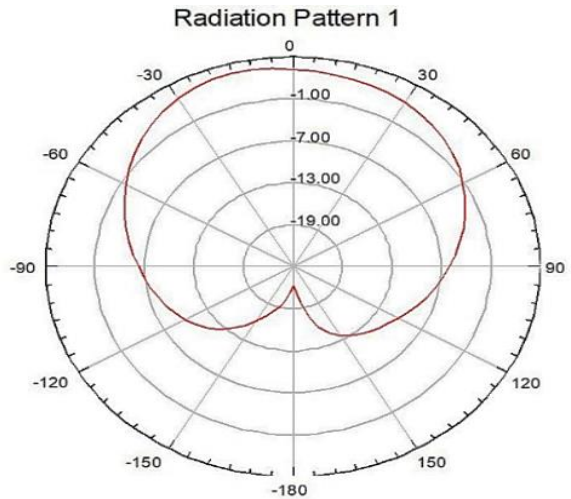

(a)

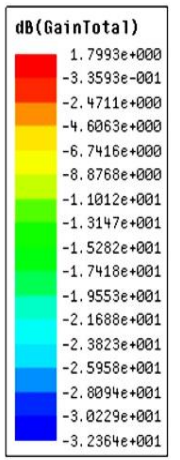

$2364 e+001$

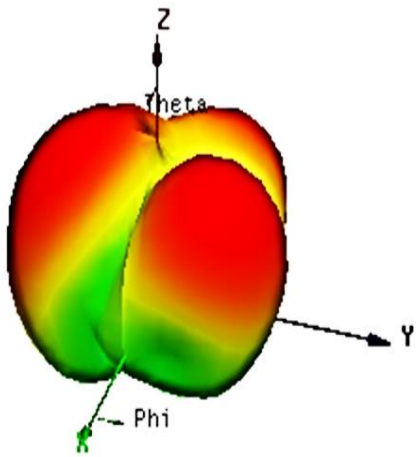

(b)

Figure 7. Simulation results of array Antenna at 2G GSM - (a) radiation pattern (b) gain

The array antenna permits further improvement in return loss and directivity from the patch. It was observed that at $2.45 \mathrm{GHz}$, compared to the theoretical array antennas with a length (Lp as in calculations) of $42.45 \mathrm{~mm}$ the optimized array with an optimal length of $-37.1 \mathrm{~dB}$ pushed the return loss to $-43.83 \mathrm{~dB}$, which once again demonstrates the superiority of the optimal design in terms of performance. A few important measurements are shown in the Table 5. Thus, this project claims the construction of high performance compact optimal antennas that are especially suited for the task of RF energy harvesting. Figure 8 shows the simulation of the Cockroft-Walton multiplier circuits, that presents the respective simulated output voltage.

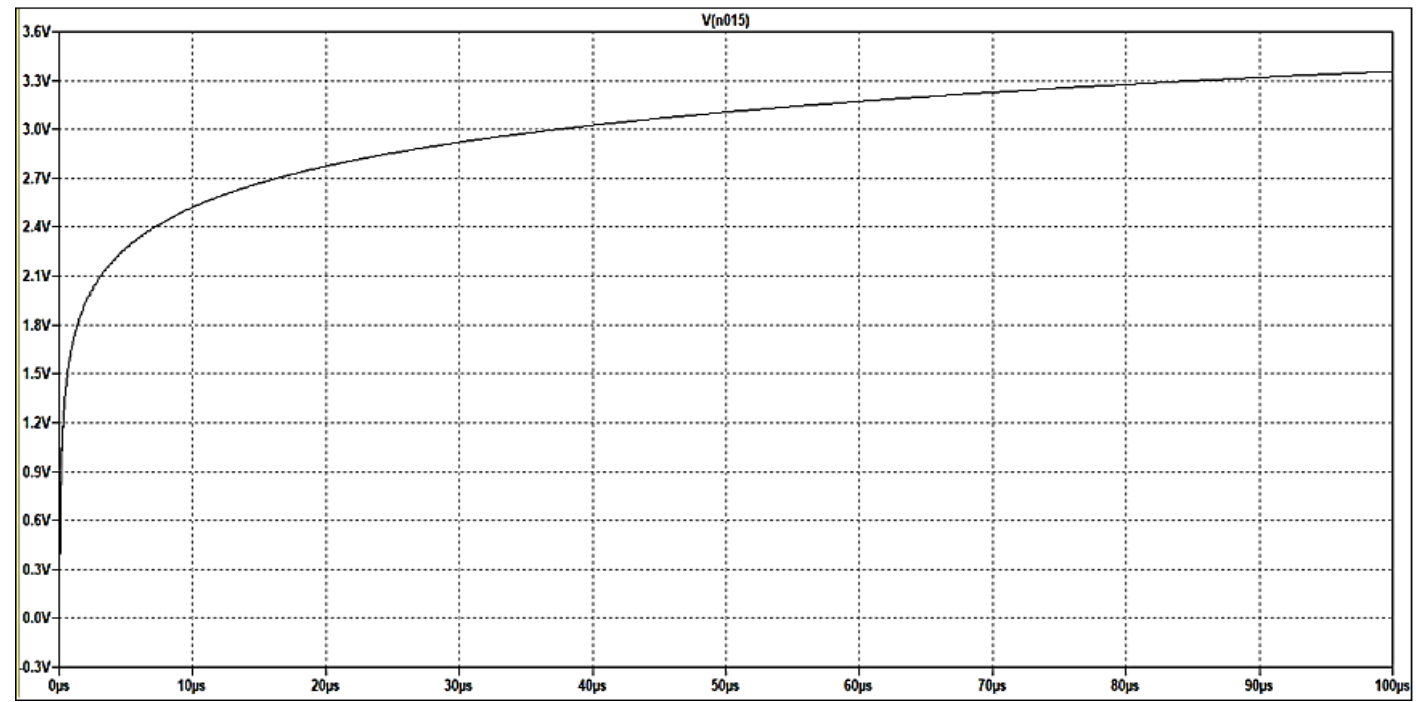

Figure 8. Simulation of charge-pump using Ansoft Designer for a 7 staged output voltage

The output voltage of finalized circuit is tested and simulated using Ansoft Designer. The simulation result of this charge-pump at 2G GSM indicating the output voltage of seven staged charge-pump circuit. All of these designs were simulated at the operating frequency of $924 \mathrm{MHz}$. With an input of $0.6 \mathrm{~V}$, the voltage multiplier managed to generate an output voltage of $3.6 \mathrm{~V} \mathrm{[20].}$

The output voltages are observed at the respective location are tabulated in the Table 6 . The values are attained with respect to the number of stages used and frequency bands. A seven staged voltage multiplier were used in this case due to certain inconsistencies faced during results verification while simulating. In order to identify the feasibility of rectification and voltage step-up effects, each category was simulated at the suggested frequencies used for harvesting. The voltages attained were considerably low in comparison to the expected output which led to further modification and alterations in the capacitance to achieve a higher output. 
Table 5. Simulation Results for the Array Antenna at Multiple RF Frequency Bands

\begin{tabular}{cccc}
\hline Name of Band & Frequency & Maximum Gain $(\mathrm{dB})$ & Return loss $(\mathrm{dB})$ \\
\hline 2G GSM & $900 \mathrm{MHz}$ & 1.6622 & -23.97 \\
3G GSM & $2.1 \mathrm{GHz}$ & 1.8209 & -22.26 \\
Wi-Fi Band 1 & $2.45 \mathrm{GHz}$ & 1.6817 & -23.52 \\
Wi-Fi Band 2 & $5 \mathrm{GHz}$ & 1.7208 & -22.88 \\
\hline
\end{tabular}

Table 6. Simulation Results of Charge-Pump at Multiple RF Frequency Bands

\begin{tabular}{cccc}
\hline Frequency & Calculated Capacitance $(\mathrm{pF})$ & \multicolumn{2}{c}{ Output Voltage } \\
\cline { 3 - 4 } & & 3 Stages & 7 Stages \\
$900 \mathrm{MHz}$ & 33 & $900 \mathrm{mV}$ & $2.5 \mathrm{~V}$ \\
$2.1 \mathrm{GHz}$ & 15 & $1 \mathrm{~V}$ & $2.4 \mathrm{~V}$ \\
$2.45 \mathrm{GHz}$ & 13 & $1.05 \mathrm{~V}$ & $2.41 \mathrm{~V}$ \\
$5 \mathrm{GHz}$ & 6 & $1.2 \mathrm{~V}$ & $2.7 \mathrm{~V}$ \\
\hline
\end{tabular}

\section{CONCLUSION}

This paper shows the advent and plausibility of employing RF energy harvesting system. This system enables wireless charging in any location at any time without restriction. The system is developed with dedicated optimal omnidirectional antenna, matching and charge pump circuit for effective signal harvesting. Four major conclusions can be drawn from the results portrayed in this work. First, the amount of power more than $-30 \mathrm{~dB}$ can be captured relatively easier irrespective of place and time. Secondly, system supports to generate a voltage more than 0.6 volts conveniently. Thirdly, the use of arrays of patch antennas in conjunction with optimization, exhibits much better return loss and gain metrics. The optimal patch antenna portrays extremely encouraging behavior and superior performance compared to many presented in literature. Finally, it is observed that Cockroft-Walton charge-pump is capable of boosting the voltage supplied by the antenna to levels that are required for practical usage. Further development is to make this harvester circuit inbuilt with the mobile device which would enable an automated charging and discharging capability and also indirectly eliminating the need of plugging in and out.

\section{REFERENCES}

[1] Hossain, Ekram, and Monowar Hasan. "5G Cellular: Key Enabling Technologies and Research Challenges." IEEE Instrumentation and Measurement Magazine. 2015; 18(3):11-21.

[2] Jabbar, Hamid, Young S. Song, and Taikyeong Ted Jeong. "RF Energy Harvesting System and Circuits For Charging of Mobile Devices." IEEE Transactions on Consumer Electronics. 2010; 56(1).

[3] Chandrasekharan Nataraj, Sheroz Khan, Mohamed. H. Habaebi, and Asan. G. A. Muthalif. "Resonant Coils Analysis for Inductively Coupled Wireless Power Transfer Applications". IEEE International Instrumentation and Measurement Technology Conference (I2MTC 2016) Proceedings. 2016; (1): 109-114.

[4] Atika Arshad, Sheroz Khan, A.H.M Zahirul Alam, Teddy S. Gunawan, and Chandrasekharan Nataraj. "An Activity Monitoring System for Senior Citizens Living Independently Using Capacitive Sensing Technique." IEEE International Instrumentation and Measurement Technology Conference (I2MTC 2016) Proceedings. 2016; (1): 449-454.

[5] Chandrasekharan Nataraj, Sheroz Khan, Mohammed Hadi Habaebi, Asan G.A. Muthalif and Ravi Lakshmanan. "Development of Low Power Wireless Power Transfer System Using Resonance Principle with Security Features". IIUM Engineering Journal (IIUMEJ). 2017; 18(2):117-127.

[6] Tan Linlin, Qiang Hao, Huang Xueliang, Cao Weijie, and Sun Wenhui. “A Novel Optimization Means of Transfer Efficiency for Resonance Coupled Wireless Power Transfer". TELKOMNIKA Indonesian Journal of Electrical Engineering. 2013; 11(5): 2747-2752.

[7] Hongwei Li, Ganrong Peng. "Energy Harvesting Device Based on Spatial Electric Field in Substations". TELKOMNIKA Indonesian Journal of Electrical Engineering. 2014; 12(6): 4771-4777.

[8] Ando, Bruno, Salvatore Baglio, Vincenzo Marletta, Antonio Pistorio, and Adi R. Bulsara. "Performance Investigation of a Nonlinear Energy Harvester with Random Vibrations and Subthreshold Deterministic Signals". IEEE Transactions on Instrumentation and Measurements. 2017; 66(5): 992-1001.

[9] Porto, Rodrigo Wolff, Valner J. Brusamarello, Ivan Müller, Fabian Leonardo Cabrera Riano, and Fernando Rangel De Sousa. "Wireless Power Transfer for Contactless Instrumentation and Measurement." IEEE Instrumentation \& Measurement Magazine. 2017; 20(4): 49-54.

[10] Yen Kheng Tan, and Sanjib Kumar Panda. "Energy Harvesting From Hybrid Indoor Ambient Light and Thermal Energy Sources for Enhanced Performance of Wireless Sensor Nodes." IEEE Transactions on Industrial Electronics. 2011; 58(9):4424 - 4435.

[11] Ali Q. "Design \& Implementation af A Mobile Phone Charging System Based on Solar Energy Harvesting." 1st International Conference on Energy, Power and Control (EPC-IQ). 2010; Basrah, Iraq. 
[12] Yap Y, Naayagi R, and Woo W. "Thermoelectric Energy Harvesting for Mobile Phone Charging Application." IEEE Region 10 Conference (TENCON). 2016.

[13] Ajay S, and Jegadhish Kumar K. "A Highly Efficient Power Management System for Charging Mobile Phones using RF Energy Harvesting." International Journal of Information Technology Convergence and Services. $2011 ; 1(5)$

[14] Pinuela M, Mitcheson P, and Lucyszyn S. "Ambient RF Energy Harvesting in Urban and Semi-Urban Environments." IEEE Transactions on Microwave Theory and Techniques. 2013; 61(7).

[15] Danilo De Donno, Luca Catarinucci, and Luciano Tarricone. "An UHF RFID Energy-Harvesting System Enhanced by a DC-DC Charge Pump in Silicon-on-Insulator Technology." IEEE Microwave and Wireless Components Letters. 2013; 23(6):315-317.

[16] Harrist. "Wireless Battery Charging System using Radio Frequency Energy Harvesting." The School of Engineering in partial fulfilment of the requirements for the degree of Master of Science. 2001; Pittsburgh.

[17] Hina Tabassum, Ekram Hossain, Md. Jahangir Hossain, and Dong in Kim. "On the Spectral Efficiency of Multiuser Scheduling in RF-Powered Uplink Cellular Networks." IEEE Transactions on Wireless Communications. 2015; 14(7): 3586 - 3600.

[18] Balanis. "Antenna Theory: Analysis and Design.” 2015; 3rd edition, John Wiley \& Sons.

[19] Maennel C. "Improvement in the modelling of a half-wave Cockroft-Walton voltage multiplier." Review of Scientific Instruments. 2013; 84(6):064701.

[20] RTL-SDR. "High Performance Low Power Advance Digital TV Silicon Tuner Datasheet." Available at:http://rtlsdr.com/wp-content/uploads/2013/04/R820T_R20111130_unlocked.pdf. 2017. 